\title{
A wait-and-watch approach to small pancreatic neuroendocrine tumors: prognosis and survival
}

\author{
Sara Massironi ${ }^{1}$, Roberta Elisa Rossi ${ }^{1,2}$, Alessandra Zilli ${ }^{1,2}$, Giovanni Casazza ${ }^{3}$, \\ Clorinda Ciafardini ${ }^{1}$, Dario Conte ${ }^{1,2}$ \\ ${ }^{1}$ Gastroenterology and Endoscopy Unit, Fondazione IRCCS Ca' Granda Ospedale Maggiore Policlinico, Milan, Italy \\ ${ }^{2}$ Postgraduate School of Gastroenterology, Department of Pathophysiology and Transplantation, Università degli Studi di \\ Milano, Milan, Italy \\ ${ }^{3}$ Department of Biomedical and Clinical Sciences "L. Sacco", University of Milan, Milan, Italy \\ Correspondence to: Sara Massironi, email: sara.massironi@policlinico.mi.it
}

Keywords: pancreatic neuroendocrine neoplasms, neuroendocrine tumors, pNEN, non-functional pancreatic neuroendocrine tumors

Received: December 22, $2015 \quad$ Accepted: February 11, $2016 \quad$ Published: March 3, 2016

\section{ABSTRACT}

Background: Whether all the small $(\varnothing \leq 20 \mathrm{~mm})$ non-functional pancreatic neuroendocrine neoplasms ( $\mathrm{PNENs}$ ) should be routinely resected is unclear.

Aim: To assess the overall survival (OS) and progression-free survival (PFS) of patients with small pNENs, followed-up with different management options.

Material and methods: Between 2007-2014, 51 patients were newly diagnosed with pNEN. 15 patients with pNENs $\varnothing \leq 20 \mathrm{~mm}$ underwent an intensive followup at 3-month intervals during the first year and then every 6 months (FU pNEN group). They were all at TNM stage I, except for one patient at stage IIA. 21 patients underwent surgical resection (SR pNEN group): 2 patients were at TNM stage I, 9 IIA, one IIIB, 9 IV. 15 patients received systemic therapy (ST pNEN group) due to advanced disease or contraindications to surgery: 5 were at stage IIA, 2 IIB, 8 IV.

Results: The median follow-up for the entire cohort was $\mathbf{5 0}$ months. Survival was similar in the FU and SR pNEN groups, but significantly worst in the ST pNEN patients (log-rank test $P<0.05)$. The 4-year survival rate was $100 \%$ in the FU pNEN group, $90.5 \%$ among the SR pNEN patients, $61 \%$ for the ST pNEN ones $(p<0.0001)$. The disease remained stable in all but one patient in the FU pNEN group, whereas six patients in the SR group and five in the ST group showed disease progression.

Conclusions: The "wait-and-watch" approach to early-stage small pNENs appears to be safe although further studies are needed to confirm these results in larger cohorts of patients.

\section{INTRODUCTION}

Pancreatic neuroendocrine neoplasms (pNENs) are rare neoplasms, even if their incidence is on the increase worldwide [1-3] as well documented over the last two decades [4]. As recently pointed out by SEER the incidence of pNENs with $\mathrm{a} \leq 2 \mathrm{~cm}$ size has increased by $710.4 \%$ (with an annual $12.8 \%$ change) over 22 years [5]. Such a fact may result from the increasing use of endoscopic ultrasound, which implies the greater ability to detect small pNENs.

PNENs represent ca. 2\% of all pancreatic tumors and may be either non-functioning (50\% to $90 \%)$ or functioning (10\% to $50 \%)$. PNENs are characterized by great biological variability. It has been hypothesized that larger neoplasms have a greater potential for aggressive behavior, whereas smaller $(\varnothing \leq 2 \mathrm{~cm})$, low-grade, nonfunctioning tumors usually display a more benign behavior, with slow growth and an overall good prognosis [5]. However, nodal and distant metastases as well as disease recurrence have been documented with regard to small tumors, suggesting that such tumors too may have a malignant potential [5-9]. On the other hand, good overall survival (up to $100 \%$ after 5 years) and little impact on survival from small pNENs, incidentally discovered, have been described [10-11]. In a recent bi-institutional study 
Gaujoux et al. [12] observed that none of their 46 patients with small incidentally discovered pNENs had developed distant or nodal metastases after a median follow-up of 34 months and an average number of serial imaging sessions at 4 . In 6 patients $(13 \%)$ a $\geq 20 \%$ increase in size was observed, but no patient nor tumor characteristics were found to be significant predictors of tumor growth.

Several attempts to determine factors that are predictive of tumor growth, nodal or distant metastases, or survival have been made, with inconsistent results until now.

With these areas of uncertainty in mind, surgical resection has always been considered as the most appropriate management option for these tumors because of: their heterogeneous, often unpredictable biological behavior, the lack of specific prognostic factors and data from the literature suggesting a positive effect of surgery on overall pNEN survival. In a retrospective study on 380 patients Sharpe et al. [13] demonstrated that with regard to non-functional $\varnothing \leq 2 \mathrm{~cm}$ sized pNENs surgical resection delivers a survival benefit, with a five-year overall survival (OS) of $82.2 \%$ for patients who underwent surgery and $34.3 \%$ for patients who underwent observation only. Noteworthy, the tumor size and margin status were not predictors of survival, whilst lymph node positivity was found to be associated with a decreased 5-year OS. On the other hand, a relevant morbidity $(<5 \%)$ and mortality $(40 \%-50 \%)$ is associated with pancreatic resection [1415] and long-term exocrine and endocrine pancreatic insufficiency may affect the quality of life [16].

Despite a substantial controversy regarding the best management options for pNENs smaller than $2 \mathrm{~cm}$, the European Neuroendocrine Tumor Society (ENETS) guidelines now recommend a "wait and see" policy in selected patients with small asymptomatic pNENs [17] in view of the slow growth of such tumors and good overall survival [10]. This strategy has gained acceptance for multiple endocrine neoplasia type 1 (MEN-1) patients [18-20] and some retrospective series have validated this approach also with regard to sporadic incidentally identified non-functioning pNENs smaller than $2 \mathrm{~cm}$. Lee et al. [21] observed a nearly 50\% risk of complications among the 57 patients who underwent surgery. The remaining 77 patients were conservatively managed and they were all free of disease progression, suggesting that non-operative management may be advocated for carefully selected pNENs when serial imaging demonstrates minimal or no growth without suspicious features.

However, the currently recommended conservative approach to small pNENs is based on under-powered and retrospective series and the resection of small, nonfunctioning tumors has not been compared to date with the conservative approach in prospective trials.

Based on the above reported findings, our present prospective series was aimed at evaluating both OS and progression-free survival (PFS) of patients with small pNENs evaluated at a single Institution according to the different clinical management options.

\section{RESULTS}

The median follow-up for the entire cohort was 50 months (range 2-84). The overall 4-year survival rate was $86 \%$. At the end of the study, 44 patients $(86 \%)$ were still alive. Of the 7 patients (14\%) who had died, 6 died of disease-related causes, whereas one patient passed away for unrelated causes.

The OS was similar in the FU pNEN and SR pNEN groups, but, as expected, it was significantly worse in the ST pNEN group (log-rank test $\mathrm{p}=0.018$ for the overall comparison; $\mathrm{p}=0.023$ for the comparison between the ST pNEN and the FU pNEN groups, adjusted for multiple comparisons) (Figure 1). The 4-year survival rate was $100 \%$ in the FU pNEN group, $90.5 \%$ in the SR pNEN group and $61 \%$ in the ST pNEN one, respectively (p $<0.0001$ at $\chi^{2}$ test).

Progression-free survival (PFS) did not differ among the three groups (log-rank test $\mathrm{p}=0.304)$, even if disease remained stable in all but one FU patients $(7 \%)$, whereas six patients $(28 \%)$ in the SR group and five (31\%) in the
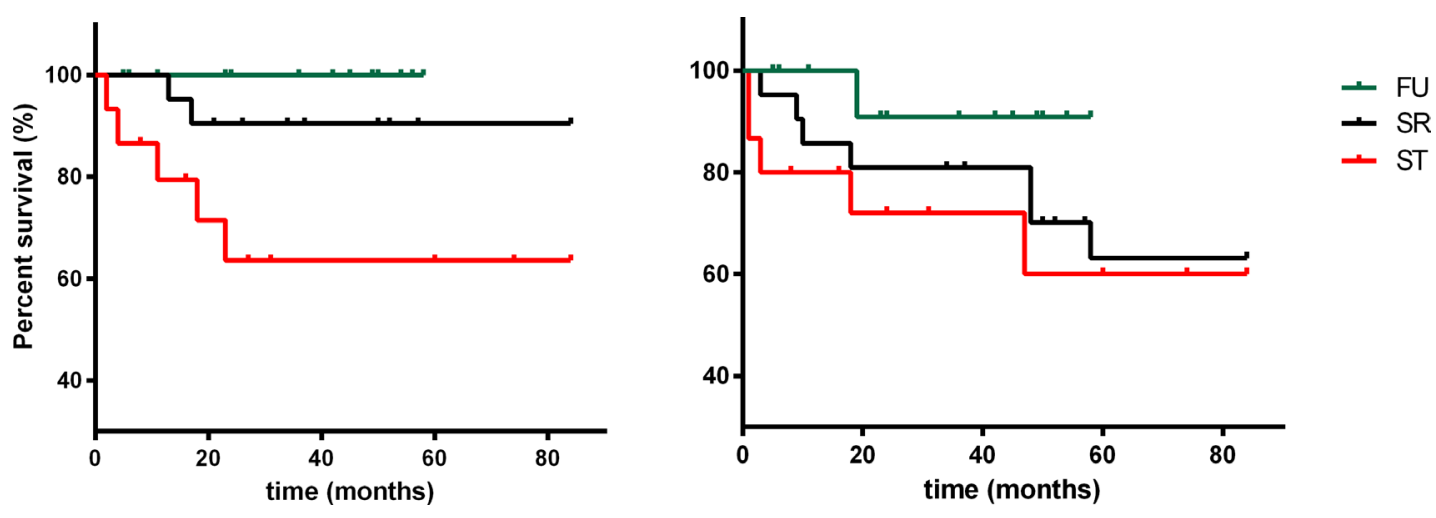

Figure 1: Overall survival (OS) and progression-free survival (PFS) of patients who have undergone follow-up (FU), surgical resection (SR) and systemic therapy (ST), respectively. 
ST group showed disease progression after 51 months follow-up.

With regard to the FU pNEN group, the patient with disease progression was a female with a MEN-1 syndrome and showed evidence of lung and nodal metastases during follow-up, 19 months after the initial diagnosis; no surgical therapy was planned after the evidence of disease progression and she underwent medical therapy by somatostatin analogues (SSAs) with disease stabilization.

Among the 21 patients of SR pNEN group we observed a nearly $50 \%$ of complications: 5 mild (postsurgical infection), 3 moderate (pancreatic fistula) and 2 severe (severe hemorrhage) events; 6 patients developed post-surgical endocrine insufficiency (i.e. diabetes). None died because of surgical intervention. In this group, 15 patients $(71 \%)$ showed no disease recurrence, whereas 6 patients $(28 \%)$ presented disease progression after a median of 14 months (range 3-58) after surgical resection: one had pancreatic recurrence and $5(83 \%)$ had hepatic metastatization. All the 6 patients with progressive disease underwent SSAs therapy; one patient was subsequently administered everolimus therapy, one patient underwent peptide receptor radionuclide therapy (PRRT) and one underwent percutaneous radiofrequency ablation of liver metastases because of further progression disease.

Regarding the ST pNEN group, 5 patients (31\%) showed disease progression, after a median of 3 months (range 1-47) after the first diagnosis. All the patients were administered second-line medical therapy, 2 patients were treated with PRRT. Four patients died from disease-related causes.

\section{DISCUSSION}

The present prospective series showed that in terms of both OS and PFS the "wait and watch" approach appears to be rational and safe when dealing with earlystage low-grade $\varnothing \leq 20 \mathrm{~mm}$ sized well-differentiated pNENs, although disease progression may occur also in this subset of patients. The optimal management option for small ( $\varnothing \leq 20 \mathrm{~mm}$ ) non-functioning $\mathrm{pNENs}$ remains to be defined. Based on several retrospective series, the ENETS guidelines now recommend a 'wait and see' policy for selected patients with small asymptomatic pNENs in view of their slow growth and good overall survival.

Our prospective study generally confirms the opportunity of a conservative approach in these small non-functioning pNENs. In fact, both OS and PFS were similar in the SR pNEN and FU pNEN groups and only one patient experienced disease progression in the FU group after 20 months from the initial diagnosis. That patient, with MEN-1 syndrome, was initially diagnosed with multiple non-functioning $\mathrm{pNENs}$, the largest being $\varnothing$ $13 \mathrm{~mm}$ sized. During the follow-up period she developed local progression, nodal and distant metastases, which controindicated a surgical approach; she was treated with SSAs and is currently alive, with stable disease. The disease progression was likely to result from the impredictable biological behavior which characterizes pNENs. The woman was included in the FU pNEN group in view of her tumor features (non-functioning tumor, $\varnothing<2 \mathrm{~cm}$, grade 1 ) and because the current management policy for MEN1 syndrome suggests a non-operative approach [18-20]. In addition, for MEN-1 patients the risk of malignancy correlates to the tumor diameter and increases substantially when its size approaches or exceeds ø $3 \mathrm{~cm}[19,25]$.

The inclusion of MEN-1 patients which may be considered a potentially confounding variable reflects our real-life clinical practice. In addition, the distribution of MEN-1 cases is homogeneous among the three patient groups, thus ruling out any effect on overall results.

Moreover, it should be considered that also the patients surgically treated may experience disease progression, as occurred in 6 patients of the present series ( 3 of them died because of the disease).

Some factors, more recently described but not analyzed in the current series, could be potentially predictive of progression, such as the FDG PET status of pancreatic lesions [26-27]. In fact, the possible selection on the basis of FDG PET would help turning patients to surgery rather than to simple follow-up. Noteworthy, the identification of prognostic factors of tumor growth would be of great help to select for observation only those patients with benign and slow-growing tumors.

The strengths of present study include: its prospective nature which is of relevance as pertinent data from randomized controlled trials are not available, the patients' homogeneous management and follow-up at a single treatment centre, and the inclusion of PFS among the primary endpoints, besides OS, which is clinically more relevant as NENs are known for their generally good prognosis. Conversely, our study presents some limits, in particular, regarding the small sample size and the different tumor stages at presentation of the three groups (Table 1), which may affect the results as the three compared groups presented different prognostic features, i.e. tumor stage and size. In particular an imbalance of prognostic patient characteristics is mainly evident among FU and SR /ST group. Furthermore, the similar survival of FU and SR group may be partially due to the less favorable prognostic features of SR group compared to FU group. However, the study design (i.e. prospective observational study) entails intrinsic limitations which could have been avoided only through a randomized trial, equally distributing the potentially confounding variables among the different groups. Anyway, such study is difficult to be designed due to both the rarity of the disease and the ethic-related issues. Meanwhile, our observational study, despite its intrinsic limitations, may provide preliminary information to support the "wait and see" policy, which is currently suggested by international guidelines and expert opinions [17], even if not properly evidence-based.

On the other hand, the comparison between SR and ST groups (well balanced per stage and $\mathrm{T}$ size), show a 
Table 1: Demographic and laboratory characteristics of patients with pancreatic neuroendocrine neoplasms (pNENs)

\begin{tabular}{lccc}
\hline Parameter & FU $(\mathbf{n}=\mathbf{1 5})$ & SR $(\mathbf{n}=\mathbf{2 1})$ & ST $(\mathbf{n}=\mathbf{1 5})$ \\
\hline Male/Female (n) & $6 / 9$ & $7 / 14$ & $7 / 8$ \\
Age, no. of years & $65(27-84)$ & $52(27-82)$ & $72(27-87)$ \\
TNM stage (no. of pts) & 14 & 2 & - \\
$\quad$ I & 1 & 9 & 7 \\
$\quad$ II & - & 1 & - \\
$\quad$ III & - & 9 & $26(20-60)$ \\
Size (mm) & $11(7-20)$ & $22(8-60)$ & $8 / 5 / 2$ \\
Grade (G1/G2/G3) & $15 / 0 / 0$ & $10 / 10 / 1$ & $5 / 10$ \\
Functioning yes/no (no. of & $-/ 15$ & $6 / 15$ & 2 \\
pts) & 3 & 5 & $27(2-84)$ \\
MEN1 (no. of cases) & $36(5-58)$ & $57(13-84)$ & $136(19-1236)$ \\
Follow-up (no. of months) & $40(12-267)$ & $28(9-3240)$ & \\
Chromogranin A (U/L) & &
\end{tabular}

Data are expressed as median (and range) unless specified otherwise.

G1: neuroendocrine tumor with Ki-67 $\leq 2 \%$; G2: neuroendocrine tumor with Ki-67 between 3 and 20\%; G3: NEN with Ki$67>20 \%$

better outcome in SR group respect to ST, confirming the positive impact of primary tumour resection on overall survival in patient in stage IV. Moreover in the FU pNEN group, the staging assessment was performed only through morphological and functional imaging, differently from the patients undergoing surgery, for which the staging was also post-surgical. In the FU pNEN group nodal involvement and/or micrometastases might have also been present and not detected by imaging techniques: this supporting the possible underestimation of disease stage in this cohort, even if in this study a worsening survival has not been observed with regard to those patients treated with a conservative approach.

Despite the reported limitations, we indeed observed that pNENs $\varnothing \leq 20 \mathrm{~mm}$ included in the FU group showed a 4-year survival rate of $100 \%$, which represents the most significant result, demonstrating the safety of such an approach.

In summary, on the basis of the present series, consideration should be given to a "wait and watch" approach to carefully selected small early-stage welldifferentiated non-functioning pNENs, although further studies comparing patients with the same prognostic characteristics in larger cohort of patients with longer follow-up periods are required in order to confirm these results.

\section{PATIENTS AND METHODS}

Between December 2007 and December 2014, 51 patients were newly diagnosed with pNENs based on clinical data, imaging (computed tomography $=\mathrm{CT}$, magnetic resonance imaging $=$ MRI, ${ }^{68}$ Gallium positron emission tomography $=$ Ga-68 PET), ultrasound endoscopy, histology. The patients had been consecutively enrolled at the Gastroenterology and Endoscopy Unit of Fondazione IRCCS Ca' Granda Ospedale Maggiore Policlinico of Milan, Italy.

The patient characteristics are detailed in Table 1. Eleven cases $(21.6 \%)$ presented a functioning tumor: 6 with gastrinoma, 2 with glucagonoma, one with insulinoma, one with VIPoma and one had a pancreatic tumor producing serotonin; the remaining 40 patients (78.4\%) had a non-functioning neoplasm. The tumors were staged according to the TMN stage scoring system [22] and classified, on the basis of their immunohistochemical characteristics according to the WHO 2010 classification, as pNENs of grade $(\mathrm{G}) 1(\mathrm{Ki}-67 \leq 2 \%), \mathrm{G} 2(\mathrm{Ki}-67 \mathrm{3}-20 \%)$ and $\mathrm{G} 3(\mathrm{Ki}-67>20 \%)$ [23].

Chromogranin A $(\mathrm{CgA})$ and specific circulating peptides were evaluated at diagnosis and during followup. CgA was measured using a commercially available kit (Dako Chromogranin A Elisa Kit, Dako A/S, Glostrup, Denmark). A regular clinical, biochemical and imaging follow-up was undertaken in all the cases (every quarter during the first year and twice a year thereafter). Morphological imaging was used to evaluate the objective responses (i.e. tumor size) according to the criteria released by the Italian Trials in Medical Oncology group [24]: classifying as complete, partial (with a tumor size decrease of $>50 \%$ ), stable (with a decrease of $<50 \%$ or an increase $25 \%$ ) and progressive (if the increase was $>25 \%$ ). 
Among the 51 patients, 15 (male/female 6/9, median age 65 years, range $27-84$ years) with pNENs $\varnothing \leq 20 \mathrm{~mm}$ were proposed for an intensive 3-month follow-up during the first year and then on 6-month intervals by CT or MRI and every 2 years by Ga-68 PET (FU pNEN group). They were all at stage I according to the TNM classification, except for one patient (stage IIA). All the patients had a well differentiated G1 pNEN. Three patients had a MEN-1 syndrome.

21 patients (male/female $7 / 14$, median age 52 years, range 27-82 years) underwent surgical resection (SR pNEN group) because of functioning neoplasms, grade $>\mathrm{G} 1$, size $\varnothing>20 \mathrm{~mm}$. Two patients were at TNM stage I, 9 at stage IIA, one at stage IIIB and 9 at stage IV. Ten patients had a G1 pNEN, 10 had a G2 pNEN and one had a G3 pNEN. Six patients $(28.6 \%$ ) had a functioning tumor; five had a MEN-1 syndrome.

The remaining 15 patients (male/female 7/8, median age 72 years, range 27-87 years) received systemic therapy (ST pNEN group) because of their advanced disease or contraindications to surgery; 5 patients were at stage IIA, 2 at stage IIB, 8 at stage IV. Eight patients had G1, 5 had G2 and 2 had G3 pNEN. Five patients had a functioning tumor, 2 had a MEN-1 syndrome.

All the patients gave their written informed consent to the study, which had been approved by the local Ethics Committee.

\section{Statistical analysis}

All the continuous variables are reported as median and range unless stated otherwise. Continuous data were analyzed using the non-parametric Mann-Whitney test. Differences between percentages were evaluated by $\chi 2$ test.

The survival curves were estimated using the Kaplan-Meier method and the log-rank test was used to compare the survival curves between different patient groups.

Statistical analyses were performed using MedCalc sotware and a p value less than 0.05 was considered statistically significant.

\section{ACKNOWLEDGMENTS} revision

We thank Marcello Hinxman for English language

\section{CONFLICTS OF INTEREST}

The authors declare no conflicts of interest.

\section{REFERENCES}

1. Yao JC, Phan AT, Jehl V, Shah G, Meric-Bernstam F. Everolimus in advanced pancreatic neuroendocrine tumors: the clinical experience. Cancer Res. 2013; 73: 1449-53. doi: 10.1158/0008-5472.CAN-12-3923. PMID: 23436795.

2. Franko J, Feng W, Yip L, Genovese E, Moser A. Nonfunctional neuroendocrine carcinoma of the pancreas: incidence, tumor biology, and outcomes in 2,158 patients. $\mathrm{J}$ Gastrointest Surg. 2010; 14:541-8.

3. Korse C.M., Taal B.G., van Velthuysen M.F., Visser O. Incidence and survival of neuroendocrine tumours in the Netherlands according to histological grade: experience of two decades of cancer registry. European Journal of Cancer. 2013; 49:1975-1983.

4. Lawrence B, Gustafsson BI, Chan A, Svejda B, Kidd M, Modlin IM. The epidemiology of gastroenteropancreatic neuroendocrine tumors. Endocrinol Metab Clin North Am. 2011; 40:1-18, vii. doi: 10.1016/j.ecl.2010.12.005. PMID: 21349409.

5. Kuo EJ, Salem RR. Population-level analysis of pancreatic neuroendocrine tumors $2 \mathrm{~cm}$ or less in size. Ann Surg Oncol. 2013; 20:2815-21. doi:10.1245/s10434-013-30057. PMID: 23771245.

6. Falconi M, Zerbi A, Crippa S, Balzano G, Boninsegna L, Capitanio V, Bassi C, Di Carlo V, Pederzoli P. Parenchymapreserving resections for small nonfunctioning pancreatic endocrine tumors. Ann Surg Oncol. 2010; 17:1621-7. doi: 10.1245/s10434-010-0949-8. PMID:20162460.

7. Vagefi PA, Razo O, Deshpande V, McGrath DJ, Lauwers GY, Thayer SP, Warshaw AL, Fernández-Del Castillo C. Evolving patterns in the detection and outcomes of pancreatic neuroendocrine neoplasms: the Massachusetts General Hospital experience from 1977 to 2005. Arch Surg. 2007; 142:347-54. PMID: 17438169; PMCID: PMC3979851.

8. Klöppel G. Classification and pathology of gastroenteropancreatic neuroendocrine neoplasms. Endocr Relat Cancer. 2011; 18:S1-16. doi: 10.1530/ERC-11-0013. PMID: 22005112.

9. Cherenfant J, Stocker SJ, Gage MK, Du H, Thurow TA, Odeleye M, Schimpke SW, Kaul KL, Hall CR, Lamzabi I, Gattuso P, Winchester DJ, Marsh RW, et al. Predicting aggressive behavior in nonfunctioning pancreatic neuroendocrine tumors. Surgery. 2013; 154:785-91; discussion 791-3. doi: 10.1016/j.surg.2013.07.004. PMID: 24074416

10. Bettini R, Partelli S, Boninsegna L, Capelli P, Crippa S, Pederzoli P, Scarpa A, Falconi M. Tumor size correlates with malignancy in nonfunctioning pancreatic endocrine tumor. Surgery. 2011; 150:75-82. doi: 10.1016/j. surg.2011.02.022. PMID: 21683859.

11. Cheema A, Weber J, Strosberg JR. Incidental detection of pancreatic neuroendocrine tumors: an analysis of incidence and outcomes. Ann Surg Oncol. 2012; 19:2932-6. doi: 10.1245/s10434-012-2285-7. PMID: 22350605.

12. Gaujoux S, Partelli S, Maire F, D'Onofrio M, Larroque B, Tamburrino D, Sauvanet A, Falconi M, Ruszniewski P. 
Observational study of natural history of small sporadic nonfunctioning pancreatic neuroendocrine tumors. J Clin Endocrinol Metab. 2013; 98:4784-9. doi: 10.1210/jc.20132604. PMID: 24057286.

13. Sharpe SM, In H, Winchester DJ, Talamonti MS, Baker MS. Surgical resection provides an overall survival benefit for patients with small pancreatic neuroendocrine tumors. J Gastrointest Surg. 2015; 19:117-23; discussion 123. doi: 10.1007/s11605-014-2615-0. PMID: 25155459.

14. Büchler MW, Wagner M, Schmied BM, Uhl W, Friess $\mathrm{H}, \mathrm{Z}$ 'graggen $\mathrm{K}$. Changes in morbidity after pancreatic resection: toward the end of completion pancreatectomy. Arch Surg. 2003; 138:1310-4; discussion 1315. doi: 10.1001/archsurg.138.12.1310. PMID: 14662530.

15. Yeo CJ, Cameron JL, Sohn TA, Lillemoe KD, Pitt HA, Talamini MA, Hruban RH, Ord SE, Sauter PK, Coleman J, Zahurak ML, Grochow LB, Abrams RA. Six hundred fifty consecutive pancreaticoduodenectomies in the 1990s: pathology, complications, and outcomes. Ann Surg. 1997; 226:248-57. doi:10.1097/00000658-199709000-00004. PMID:933993; PMCID: PMC1191017.

16. Falconi M, Mantovani W, Crippa S, Mascetta G, Salvia R, Pederzoli P. Pancreatic insufficiency after different resections for benign tumours. Br J Surg. 2008; 95:85-91. PMID: 18041022.

17. Falconi M, Bartsch DK, Eriksson B, Klöppel G, Lopes JM, O'Connor JM, Salazar R, Taal BG, Vullierme MP, O'Toole D; Barcelona Consensus Conference participants. ENETS Consensus Guidelines for the management of patients with digestive neuroendocrine neoplasms of the digestive system: well-differentiated pancreatic non-functioning tumors. Neuroendocrinology. 2012; 95:120-34. doi:10.1159/000335587. PMID: 22261872. doi:10.1159/000335587.

18. Triponez F, Dosseh D, Goudet P, Cougard P, Bauters C, Murat A, Cadiot G, Niccoli-Sire P, Chayvialle JA, Calender A, Proye CA. Epidemiology data on 108 MEN1 patients from the GTE with isolated nonfunctioning tumors of the pancreas. Ann Surg. 2006; 243:265-72. PMID: 16432361; PMCID: PMC1448903.

19. Triponez F, Goudet P, Dosseh D, Cougard P, Bauters C, Murat A, Cadiot G, Niccoli-Sire P, Calender A, Proye CA; French Endocrine Tumor Study Group. Is surgery beneficial for MEN1 patients with small $(<$ or $=2 \mathrm{~cm})$, nonfunctioning pancreaticoduodenal endocrine tumor? An analysis of 65 patients from the GTE. World J Surg. 2006; 30:654-62; discussion 663-4. PMID: 16680582.

20. Kann PH, Balakina E, Ivan D, Bartsch DK, Meyer S, Klose KJ, Behr T, Langer P. Natural course of small, asymptomatic neuroendocrine pancreatic tumours in multiple endocrine neoplasia type 1: an endoscopic ultrasound imaging study. Endocr Relat Cancer. 2006; 13:1195-202. PMID: 17158764.

21. Lee LC, Grant CS, Salomao DR, Fletcher JG, Takahashi N, Fidler JL, Levy MJ, Huebner M. Small, nonfunctioning, asymptomatic pancreatic neuroendocrine tumors (PNETs): role for nonoperative management. Surgery. 2012; 152:96574. doi: 10.1016/j.surg.2012.08.038. PMID: 23102679.

22. Rindi G, Klöppel G, Alhman H, Caplin M, Couvelard A, de Herder WW, Erikssson B, Falchetti A, Falconi M, Komminoth P, Körner M, Lopes JM, McNicol AM, et al. TNM staging of foregut neuroendocrine tumors: a consensus proposal including agrading system. Virchows Arch. 2006; 449:395-401. PMID: 16967267; PMCID: PMC1888719.

23. Bosman FT: WHO Classification of Tumor of the Digestive System. Lyon, IARC Press, 2010.

24. Bajetta E, Procopio G, Catena L, Martinetti A, De Dosso S, Ricci S, Lecchi AS, Boscani PF, Iacobelli S, Carteni G, De Braud F, Loli P, Tartaglia A, et al. Lanreotide autogel every 6 weeks compared with Lanreotide microparticles every 3 weeks in patients with well differentiated neuroendocrine tumors: a Phase III Study. Cancer. 2006; 107:2474-81.

25. Kouvaraki MA, Shapiro SE, Cote GJ, Lee JE, Yao JC, Waguespack SG, Gagel RF, Evans DB, Perrier ND. Management of pancreatic endocrine tumors in multiple endocrine neoplasia type 1. World J Surg. 2006; 30:64353. PMID: 16680581.

26. Squires MH 3rd, Volkan Adsay N, Schuster DM, Russell MC, Cardona K, Delman KA, Winer JH, Altinel D, Sarmiento JM, El-Rayes B, Hawk N, Staley CA 3rd, Maithel SK, et al. Octreoscan Versus FDG-PET for Neuroendocrine Tumor Staging: A Biological Approach. Ann Surg Oncol. 2015; 22:2295-301. doi:10.1245/s10434015-4471-x. PMID: 25786743.

27. Luo G, Liu Z, Guo M, Jin K, Xiao Z, Liu L, Xu J, Zhang B, Liu C, Huang D, Hu S, Ni Q, Long J, et al. (18)F-FDG $\mathrm{PET} / \mathrm{CT}$ can be used to detect non-functioning pancreatic neuroendocrine tumors. Int J Oncol. 2014; 45:1531-6. doi: 10.3892/ijo.2014.2570. PMID: 25096059. 